\title{
Outcomes of Common Fixed Point Theorems in S-metric Space
}

\author{
Katta Mallaiah $^{1}$, Veladi Srinivas ${ }^{2, *}$ \\ ${ }^{1}$ Department of Technical Education, JNGP Ramanthapur, Telangana, India \\ ${ }^{2}$ University College of Science, Saifabad, Osmania University, Telangana, India
}

\begin{abstract}
Cite This Paper in the following Citation Styles
(a): [1] Katta Mallaiah, Veladi Srinivas, "Outcomes of Common Fixed Point Theorems in S-metric Space," Mathematics and Statistics, Vol.10, No.1, pp. 160-165,
\end{abstract} 2022. DOI: 10.13189/ms.2022.100114

(b): Katta Mallaiah, Veladi Srinivas, (2022). Outcomes of Common Fixed Point Theorems in S-metric Space. Mathematics and Statistics, 10(1), 160-165. DOI: 10.13189/ms.2022.100114

Copyright $(2022$ by authors, all rights reserved. Authors agree that this article remains permanently open access under the terms of the Creative Commons Attribution License 4.0 International License

\begin{abstract}
In the present paper, we establish the existence of two unique common fixed point theorems with a new contractive condition for four self-mappings in the S-metric space. First, we establish a common fixed-point theorem by using weaker conditions such as compatible mappings of type-(E) and subsequentially continuous mappings. Further, in the next theorem, we use another set of weaker conditions like sub-compatible and sub-sequentially continuous mappings, which are weaker than occasionally weakly compatible mappings. Moreover, it is observed that the mappings in these two theorems are sub-sequentially continuous, but these mappings are neither continuous nor reciprocally continuous mappings. These two results will extend and generalize the existing results of [7] and [9] in the S-metric space. Furthermore, we also provide some suitable examples to justify our outcomes.
\end{abstract}

Keywords S-metric Space, Coincidence Point, Compatible Mappings of Type-E ,Sub-compatible and Sub-sequential Continuous Mapping

\section{Introduction}

The Metric space theory was developed in a wide range in the field of mathematics. S-metric space emerges as a generalization of a metric space. For the past several years, the concept of commutative, compatibility, different types of compatible mappings like compatible type-A, type-B, type-C, and type-P became highly significant due to the establishment of many fixed point theorems in different spaces. Compatible mappings imply weakly compatible, but not conversely. Further, occasionally weakly compatible mappings are weaker than weakly compatible mappings. Similarly, the concept of continuity has been transformed to its weaker forms like reciprocally continuous, sub sequentially continuous mappings. Using the concept of S-metric space, we discussed certain basic definitions and examples which were useful in our main results. Sedghi.S, Shobe. N, and Aliouche.A[1] proposed the concept of S-metric space and proved Various theorems. Y.R.Singh and Mahendra Singh [2] developed the concept of compatible mapping of type- E and generalized some fixed point theorems related to it. Bouhadjera and Godet Thobie $[3],[4]$ introduced the concept of sub-sequentially continuous[5] mappings and proved many interesting results in metric space. The concept of reciprocal continuity of mappings in metric spaces was developed by Pant [6].

In this article, we propose to use the new contraction condition[7] , [8] and prove two common fixed point theorems in S-metric space, using weaker conditions like sub-sequentially continuous mappings [9] and compatible mappings of type-E. Further, we discuss another result by using sub-compatibility and subsequentially continuous mappings.

Before we prove our theorem, we'll discuss some definitions and examples.

\section{PRELIMINARIES}

Definition 2.1 [1]" Let $X$ be a non- empty set and $S: X^{3} \rightarrow$ $[0, \infty)$ be a function satisfying the axioms 
(i) $S(\alpha, \beta, \gamma) \geq 0$

(ii) $S(\alpha, \beta, \gamma)=0$ if and only if $\alpha=\beta=\gamma$

(iii) $S(\alpha, \beta, \gamma) \leq S(\alpha, \alpha, \omega)+S(\beta, \beta, \omega)+S(\gamma, \gamma, \omega)$ $\forall \alpha, \beta, \gamma, \omega \in X$.

Then $\mathrm{S}$ is called $\mathrm{S}$-metric on $\mathrm{X}$ and the pair $(X, S)$ is called a S-metric space."

Remark [7]

In S-metric space $(X, S)$ the following axioms holds,

(i) $S(\alpha, \alpha, \beta)=S(\beta, \beta, \alpha)$.

(ii) $S(\alpha, \alpha, \beta)=2 S(\alpha, \alpha, \gamma)+S(\beta, \beta, \gamma)$.

(iii) If $\left\{\alpha_{n}\right\}$ and $\left\{\beta_{n}\right\}$ such that $\lim _{k \rightarrow \infty} \alpha_{k}=\alpha$ and $\lim _{k \rightarrow \infty} \beta_{k}=\beta$ then $S\left(\alpha_{k}, \alpha_{k}, \beta_{k}\right)=S(\alpha, \alpha, \beta)$.

Definition $2.2[1]$ " We define self maps $P$ and $Q$ of an S-metric space $(X, S)$ as called compatible if $\lim _{k \rightarrow \infty} S\left(P Q \alpha_{k}, P Q \alpha_{k}, Q P \alpha_{k}\right)=0$ whenever $\exists$ a sequence $\left\{\alpha_{k}\right\} \in X$ such that $\lim _{k \rightarrow \infty} P \alpha_{k}=\lim _{k \rightarrow \infty} Q \alpha_{k}=\nu$ for all $\nu \in X$."

Definition 2.3[2] " Two mappings $\mathrm{P}$ and $\mathrm{Q}$ of an S-metric space $(X, S)$ are called compatible type- $\mathbf{E}$ if whenever $\exists$ a sequence $\left\{\alpha_{k}\right\} \in X$ such that $\lim _{k \rightarrow \infty} P \alpha_{k}=\lim _{k \rightarrow \infty} Q \alpha_{k}=\nu$ for some $\mu \in X, \lim _{k \rightarrow \infty} S\left(P^{2} \alpha_{k}, P Q \alpha_{k}, Q \nu\right)=0$ or $\lim _{k \rightarrow \infty} S\left(Q^{2} \alpha_{k}, Q P \alpha_{k}, P \nu\right)=0 . "$

Definition 2.4[6] " Two mappings P and Q of an S-metric space $(X, S)$ are called sub-sequentially continuous if whenever $\exists$ a sequence $\left\{\alpha_{k}\right\} \in X$ such that $\lim _{k \rightarrow \infty} P \alpha_{k}=\lim _{k \rightarrow \infty} Q \alpha_{k}=\nu$ for some $\nu \in X, S\left(P Q \alpha_{k}, P Q \alpha_{k}, P \nu\right)=0 \&$ $S\left(Q P \alpha_{k}, Q P \alpha_{k}, Q \nu\right)=0 . "$

Definition 2.5, [3] "Two mappings $\mathrm{P}$ and $\mathrm{Q}$ of an $\mathrm{S}$ metric space $(X, S)$ as called reciprocally continuous if whenever $\exists$ a sequence $\left\{\alpha_{k}\right\} \in X$ such that $\lim _{k \rightarrow \infty} P \alpha_{k}=\lim _{k \rightarrow \infty} Q \alpha_{k}=\nu$ for some $\nu \in X$, $S\left(P Q \alpha_{k}, P Q \alpha_{k}, P \nu\right)=0$ or $S\left(Q P \alpha_{k}, Q P \alpha_{k}, Q \nu\right)=0 . "$

\section{Now we consider an example where the} mappings are sub-sequentially continuous but neither continuous nor reciprocally continuous.

\section{Example 2.6.1}

Let $\mathrm{X}=[0,1)$ be a $\mathrm{S}$-metric space on $\mathrm{X}$ defined by $S(\alpha, \beta, \gamma)=\delta_{1}(\alpha, \gamma)+\delta_{2}(\beta, \gamma)$ where $\delta_{1}$ and $\delta_{2}$ are two metrics on $\mathrm{X}$ and define $\mathrm{P}, \mathrm{Q}$ by

$P(\alpha)=\left\{\begin{array}{ll}2-5 \alpha & \text { if } 0 \leq \alpha \leq \frac{1}{3} ; \\ 1-\alpha & \text { if } \frac{1}{3}<\alpha<1\end{array}\right.$ and
$Q(\alpha)= \begin{cases}1-2 \alpha & \text { if } 0 \leq \alpha \leq \frac{1}{3} \\ \frac{1+4 \alpha}{7} & \text { if } \frac{1}{3} \leq \alpha<1\end{cases}$

Take a sequence $\left\{\alpha_{k}\right\}$ as $\alpha_{k}=\frac{1}{3}-\frac{1}{k}$ for $k>0$.

Now $P\left(\alpha_{k}\right)=P\left(\frac{1}{3}-\frac{1}{k}\right)=2-5\left(\frac{1}{3}-\frac{1}{k}\right)=\frac{1}{3}+\frac{5}{k}=\frac{1}{3}$ and $Q\left(\alpha_{k}\right)=Q\left(\frac{1}{3}-\frac{1}{k}\right)=1-2\left(\frac{1}{3}-\frac{1}{k}\right)=\frac{1}{3}+\frac{2}{k}=\frac{1}{3}$ as $k \rightarrow \infty$.

Therefore $P\left(\alpha_{k}\right)=Q\left(\alpha_{k}\right)=\frac{1}{3}=\nu($ say $)$ as $k \rightarrow \infty$.

Also $P(\nu)=P\left(\frac{1}{3}\right)=\frac{1}{3}$ and $Q(\nu)=Q\left(\frac{1}{3}\right)=\frac{1}{3}$.

Further $P Q\left(\alpha_{k}\right)=P Q\left(\frac{1}{3}-\frac{1}{k}\right)=P\left(1-2\left(\frac{1}{3}-\frac{1}{k}\right)\right.$

$=P\left(\frac{1}{3}+\frac{2}{k}\right)=1-\left(\frac{1}{3}+\frac{2}{k}\right) \stackrel{2}{=} \frac{2}{3}$

and $Q P\left(\alpha_{k}\right)=Q P\left(\frac{1}{3}-\frac{1}{k}\right)=Q\left(2-5\left(\frac{1}{3}-\frac{1}{k}\right)\right)$

$=Q\left(\frac{1}{3}+\frac{5}{k}\right)=\frac{1+4\left(\frac{1}{3}+\frac{5}{k}\right)}{7}=\frac{1}{3}$ as $k \rightarrow \infty$.

$\therefore S\left(P Q \alpha_{k}, P Q \alpha_{k}, P \nu\right)=S\left(\frac{2}{3}, \frac{2}{3}, \frac{1}{3}\right) \neq 0$

and $S\left(Q P \alpha_{k}, Q P \alpha_{k}, Q \nu\right)=S\left(\frac{1}{3}, \frac{1}{3}, \frac{1}{3}\right)=0$

for some $\nu \in X$ as $k \rightarrow \infty$.

This shows that $\mathrm{P}$ and $\mathrm{Q}$ are not reciprocally continuous.

Now take another sequence $\left\{\beta_{k}\right\}$ as $\beta_{k}=\frac{6}{11}+\frac{1}{k}$ for $k>0$.

Now $P\left(\beta_{k}\right)=P\left(\frac{6}{11}+\frac{1}{k}\right)=1-\left(\frac{6}{11}+\frac{1}{k}\right)=\frac{5}{11}-\frac{1}{k}=\frac{5}{11}$

and $Q\left(\beta_{k}\right)=Q\left(\frac{6}{11}+\frac{1}{k}\right)=\frac{1+4\left(\frac{6}{11}+\frac{1}{k}\right)}{7}=\frac{5}{11}+\frac{4}{7 k}=\frac{5}{11}$

as $k \rightarrow \infty$.

$\therefore P\left(\beta_{k}\right)=Q\left(\beta_{k}\right)=\frac{5}{11}=\phi($ say $)$ as $k \rightarrow \infty$.

Also $P(\phi)=P\left(\frac{5}{11}\right)=\frac{6}{11}$ and $Q(\phi)=Q\left(\frac{5}{11}\right)=\frac{6}{11}$.

Further $P Q\left(\beta_{k}\right)=P Q\left(\frac{6}{11}+\frac{1}{k}\right)=P\left(\frac{1+4\left(\frac{6}{11}+\frac{1}{k}\right)}{7}\right)$

$=P\left(\frac{5}{11}+\frac{4}{7 k}\right)=1-\left(\frac{5}{11}+\frac{4}{7 k}\right)=\frac{6}{11}$

and $Q P\left(\beta_{k}\right)=Q P\left(\frac{6}{11}+\frac{1}{k}\right)=Q\left(1-\left(\frac{6}{11}+\frac{1}{k}\right)\right)$

$=Q\left(\frac{5}{11}-\frac{1}{k}\right)=\frac{1+4\left(\frac{5}{11}+\frac{5}{k}\right)}{7}=\frac{6}{11}$ as $k \rightarrow \infty$.

$\therefore S\left(P Q \beta_{k}, P Q \beta_{k}, P \nu\right)=S\left(\frac{6}{11}, \frac{6}{11}, \frac{6}{11}\right)=0$ and $S\left(Q P \beta_{k}, Q P \beta_{k}, Q \nu\right)=S\left(\frac{6}{11}, \frac{6}{11}, \frac{6}{11}\right)=0$

for some $\phi \in X$ as $k \rightarrow \infty$.

Hence $\mathrm{P}$ and $\mathrm{Q}$ are sub sequentially continuous .

Definition 2.6[9]" Two self maps $P$ and Q on a S-metric space $(\mathrm{X}, \mathrm{S})$ are said to be sub-compatible if and only if $\exists$ a sequence $\left\{\alpha_{k}\right\}$ in $\mathrm{X}$ such that $\lim _{k \rightarrow \infty} P \alpha_{k}=\lim _{k \rightarrow \infty} Q \alpha_{k}=\nu$ where $\nu \in X$ and satisfy $\lim _{k \rightarrow \infty} S\left(P Q \alpha_{k}, Q P \alpha_{k}, \nu\right)=1$ for all $\nu>0 "$.

\section{Main results}

\subsection{Theorem}

Let $\mathrm{P}, \mathrm{R}, \mathrm{Q}$ and $\mathrm{T}$ be mappings in a complete S-metric space $(X, S)$ into itself holding the conditions

(3.1.1) $P(X) \subseteq T(X)$ and $R(X) \subseteq Q(X)$ (3.1.2)

$$
\begin{gathered}
S(P \alpha, P \alpha, R \beta) \leq \lambda \max \{S(Q \alpha, Q \alpha, T \beta), S(R \beta, R \beta, P \alpha), \\
\left.\frac{S(P \alpha, P \alpha, Q \alpha) S(R \beta, R \beta, T \beta)}{S(Q \alpha, Q \alpha, T \beta)}\right\}
\end{gathered}
$$

$\exists \lambda \in(0,1)$ such that, for all $\alpha, \beta \in X$.

( 3.1.3) The pairs $(P, Q)$ and $(R, T)$ are compatible of type-E and sub-sequentially continuous mappings.

(3.1.4) The pairs $(P . Q)$ and $(R, T)$ have a coincidence point. Then $\mathrm{P}, \mathrm{R}, \mathrm{Q}$ and $\mathrm{T}$ all have a unique common fixed point in $\mathrm{X}$. Proof:

Since $P(X) \subseteq T(X)$, there is a point $\alpha_{0} \in X$ such that $P \alpha_{0}=$ $T \alpha_{1}=\beta_{0}$,for $\alpha_{1} \in X$. For this point $\alpha_{1} \exists$ a point $\alpha_{2} \in X$ such that $R \alpha_{1}=Q \alpha_{2}=\beta_{1}$.

Similarly in this way, we construct a sequence $\left\{\beta_{k}\right\} \in X$ such 
that $\beta_{2 k}=P \alpha_{2 k}=T \alpha_{2 k+1}$ and $\beta_{2 k+1}=R \alpha_{2 k+1}=Q \alpha_{2 k+2}$ for $k \geq 0$.

We have to show that $\left\{\beta_{k}\right\}$ is a cauchy sequence in S-metric space in X.

$$
\begin{aligned}
& S\left(\beta_{2 k}, \beta_{2 k}, \beta_{2 k+1}\right)=S\left(P \alpha_{2 k}, P \alpha_{2 k}, R \alpha_{2 k+1}\right) \leq \lambda \max \\
& \left\{S\left(Q \alpha_{2 k}, Q \alpha_{2 k}, T \alpha_{2 k+1}\right), S\left(R \alpha_{2 k+1}, R \alpha_{2 k+1}, P \alpha_{2 k}\right),\right. \\
& \left.\frac{S\left(P \alpha_{2 k}, P \alpha_{2 k}, Q \alpha_{2 k}\right) S\left(R \alpha_{2 k+1}, R \alpha_{2 k+1}, T \alpha_{2 k+1}\right)}{S\left(Q \alpha_{2 k}, Q \alpha_{2 k}, T \alpha_{2 k+1}\right)}\right\}
\end{aligned}
$$

this gives

$$
\begin{aligned}
& S\left(\beta_{2 k}, \beta_{2 k}, \beta_{2 k+1}\right) \leq \lambda \max \left\{S\left(\beta_{2 k-1}, \beta_{2 k-1}, \beta_{2 k}\right),\right. \\
& \left.S\left(\beta_{2 k}, \beta_{2 k}, \beta_{2 k+1}\right), \frac{S\left(\beta_{2 k}, \beta_{2 k}, \beta_{2 k-1}\right) S\left(\beta_{2 k+1}, \beta_{2 k+1}, \beta_{2 k}\right)}{S\left(\beta_{2 k-1}, \beta_{2 k-1}, \beta_{2 k}\right)}\right\}
\end{aligned}
$$

on simplification

$$
S\left(\beta_{2 k}, \beta_{2 k}, \beta_{2 k+1}\right) \leq \lambda S\left(\beta_{2 k-1}, \beta_{2 k-1}, \beta_{2 k}\right) .
$$

By similar we have

$$
S\left(\beta_{2 k-1}, \beta_{2 k-1}, \beta_{2 k}\right) \leq \lambda S\left(\beta_{2 k-2}, \beta_{2 k-2}, \beta_{2 k-1}\right) .
$$

Now from (1) and (2) we have

$S\left(\beta_{k}, \beta_{k}, \beta_{k-1}\right) \leq \lambda S\left(\beta_{k-1}, \beta_{k-1}, \beta_{k-2}\right)$

Which implies that

$$
\begin{gathered}
S\left(\beta_{k}, \beta_{k}, \beta_{k-1}\right) \leq \lambda^{k-1} S\left(\beta_{1}, \beta_{1}, \beta_{0}\right) . \\
S\left(\beta_{k}, \beta_{k}, \beta_{l}\right) \leq 2 S\left(\beta_{l}, \beta_{l}, \beta_{l+1}\right)+2 S\left(\beta_{l+1}, \beta_{l+1}, \beta_{l+2}\right) \\
+2 S\left(\beta_{l+2}, \beta_{l+2}, \beta_{l+3}\right)+\cdots+2 S\left(\beta_{k-1}, \beta_{k-1}, \beta_{k}\right) .
\end{gathered}
$$

From (4) and $0<\lambda<1$

$S\left(\beta_{k}, \beta_{k}, \beta_{l}\right) \leq 2\left(\lambda^{l}+\lambda^{l+1}+\lambda^{l+2}+\cdots+\lambda^{k-1}\right) S\left(\beta_{1}, \beta_{1}, \beta_{0}\right)$

which gives

$S\left(\beta_{k}, \beta_{k}, \beta_{l}\right) \leq 2 \lambda^{l}\left(1+\lambda+\lambda^{2}+\cdots\right) S\left(\beta_{1}, \beta_{1}, \beta_{0}\right)$

this in turn implies

$S\left(\beta_{k}, \beta_{k}, \beta_{l}\right) \leq 2 \frac{\lambda^{l}}{1-\lambda} S\left(\beta_{1}, \beta_{1}, \beta_{0}\right) \rightarrow 0$ as $k, l \rightarrow \infty$.

This results $\left\{\beta_{k}\right\}$ as a cauchy sequence in S-metric space in X.

By the completeness of $\mathrm{X}, \exists \nu$ in X such that $\lim _{k \rightarrow \infty}\left\{\beta_{k}\right\}=\nu$.

Consequently, the sub sequences $\mathrm{P} \alpha_{2 k}, \mathrm{Q} \alpha_{2 k}, \mathrm{~T} \alpha_{2 k+1}$ and

$\mathrm{R} \alpha_{2 k+1}$ of $\left\{\beta_{k}\right\}$ also converge to the same point $\nu \in X$.

Since the pair $(P, Q)$ is sub-sequentially continuous, there exists a sequence $\alpha_{k}$ in $X$ such that

$\lim _{k \rightarrow \infty} P \alpha_{k}=\lim _{k \rightarrow \infty} Q \alpha_{k}=\nu$ for some $\nu \in X$

such that

$$
S\left(P Q \alpha_{k}, P Q \alpha_{k}, P \nu\right)=0 \text { and } S\left(Q P \alpha_{k}, Q P \alpha_{k}, Q \nu\right)=0 .
$$

Since the pair $(P, Q)$ is compatible mapping of type-E, for the sequence $\alpha_{k} \in X$ such that $\lim _{k \rightarrow \infty} P \alpha_{k}=\lim _{k \rightarrow \infty} Q \alpha_{k}=\nu$ for some $\nu \in X$ such that

$S\left(P P \alpha_{k}, P Q \alpha_{k}, Q \nu\right)=0$ and $S\left(Q Q \alpha_{k}, Q P \alpha_{k}, P \nu\right)=0$.
From (5) and (6) we have

$$
P \nu=Q \nu
$$

Again since the pair $(R, T)$ is sub-sequentially continuous for the sequence $\alpha_{k} \in X$ such that $R \alpha_{k}=T \alpha_{k}=\phi($ say $)$ as $k \rightarrow \infty$ for some $\phi \in X$ such that

$$
S\left(R T \alpha_{k}, R T \alpha_{k}, R \phi\right)=0 \text { and } S\left(T R \alpha_{k}, T R \alpha_{k}, T \phi\right)=0
$$

Also the pair $(R, T)$ is compatible mapping of type-E, then there exists a sequence $\alpha_{k} \in X$ such that $R \alpha_{k}=T \alpha_{k}=\phi$ as $k \rightarrow \infty$ for some $\phi \in X$ such that

$$
S\left(R R \alpha_{k}, R T \alpha_{k}, T \phi\right)=0 \text { and } S\left(T T \alpha_{k}, T R \alpha_{k}, R \phi\right)=0
$$

From (8) and (9) we have

$$
R \phi=T \phi
$$

Hence $\nu$ is a coincidence point of $\mathrm{P} \& \mathrm{Q}$ and $\phi$ is a coincidence point of $\mathrm{R}, \mathrm{T}$.

We will show that $\nu=\phi$.

By putting $\alpha=\alpha_{k} \& \beta=\beta_{k}$ in (3.1.2) we get

$$
\begin{aligned}
& S\left(P \alpha_{k}, P \alpha_{k}, R \beta_{k}\right) \leq \\
& \lambda \max \left\{S\left(Q \alpha_{k}, Q \alpha_{k}, T \beta_{k}\right), S\left(R \beta_{k}, R \beta_{k}, P \alpha_{k}\right),\right. \\
& \left.\frac{S\left(P \alpha_{k}, P \alpha_{k}, Q \alpha_{k}\right) S\left(R \beta_{k}, R \beta_{k}, T \beta_{k}\right)}{S\left(Q \alpha_{k}, Q \alpha_{k}, T \beta_{k}\right)}\right\}
\end{aligned}
$$

letting $k \rightarrow \infty$ and using $P \alpha_{k}=Q \alpha_{k}=\nu$ and $R \beta_{k}=T \beta_{k}=\phi$

$$
\begin{aligned}
S(\nu, \nu, \phi) \leq \lambda \max & \{S(\nu, \nu, \phi), S(\phi, \phi, \nu), \\
& \left.\frac{S(\nu, \nu, \nu) S(\phi, \phi, \phi)}{S(\nu, \nu, \phi)}\right\}
\end{aligned}
$$

this in turn implies

$S(\nu, \nu, \phi) \leq \lambda \max \left\{S(\nu, \nu, \phi), S(\phi, \phi, \nu), \frac{1}{S(\nu, \nu, \phi)}\right\}$

this gives

$S(\nu, \nu, \phi) \leq \lambda S(\nu, \nu, \phi)$

$$
\nu=\phi .
$$

From (7), (10) and (11) we have

$$
P \nu=Q \nu \text { and } R \nu=T \nu .
$$

By putting $\alpha=\nu$ and $\beta=\beta_{k}$ in (3.1.2) we get

$$
\begin{aligned}
& S\left(P \nu, P \nu, R \beta_{k}\right) \leq \lambda \max \left\{S\left(Q \nu, Q \nu, T \beta_{k}\right), S\left(R \beta_{k}, R \beta_{k}, P \nu\right),\right. \\
&\left.\frac{S(P \nu, P \nu, Q \nu) S\left(R \beta_{k}, R \beta_{k}, T \beta_{k}\right)}{S\left(Q \nu, Q \nu, T \beta_{k}\right)}\right\}
\end{aligned}
$$


using $P \nu=Q \nu$ and $R \beta_{k}=T \beta_{K}=\phi$ as $k \rightarrow \infty$

$$
\begin{aligned}
S(P \nu, P \nu, \phi) \leq \lambda \max & \{S(P \nu, P \nu, \phi), S(\phi, \phi, P \nu), \\
& \left.\frac{S(\phi, \phi, P \nu) S(\phi, \phi, \phi)}{S(P \nu, P \nu, \phi)}\right\}
\end{aligned}
$$

in turns implies $S(P \nu, P \nu, \phi) \leq \lambda\{S(P \nu, P \nu, \phi)\}$

this gives $\mathrm{P} \nu=\phi$

and which implies $\mathrm{P} \nu=\nu$.

$$
\therefore P \nu=Q \nu=\nu \text {. }
$$

Replace $\alpha=\alpha_{k}$ and $\beta=\phi$ in (3.1.2) we get $S\left(P \alpha_{k}, P \alpha_{k}, R \phi\right) \leq \lambda \max \left\{S\left(Q \alpha_{k}, Q \alpha_{k}, T \phi\right), S\left(R \phi, R \phi, P \alpha_{k}\right)\right.$ $\left.\frac{S\left(P \alpha_{k}, P \alpha_{k}, Q \alpha_{k}\right) S(R \phi, R \phi, T \phi)}{S\left(Q \alpha_{k}, Q \alpha_{k}, T \phi\right)}\right\}$

using $R \phi=T \phi$ and $P \alpha_{k}=Q \alpha_{k}=\nu$ on letting $\mathrm{k} \rightarrow \infty$

$$
\begin{aligned}
S(\nu, \nu, R \phi) \leq \lambda \max & \{S(\nu, \nu, R \phi), S(R \phi, R \phi, \nu), \\
& \left.\frac{S(R \phi, R \phi, \nu) S(R \phi, R \phi, R \phi)}{S(\nu, \nu, R \phi)}\right\}
\end{aligned}
$$

this implies

$S(\nu, \nu, R \phi) \leq \lambda S(\nu, \nu, R \phi)$

which implies $R \phi=\nu$

$\Longrightarrow R \phi=\phi$.

$$
\therefore R \nu=T \nu=\nu \text {. }
$$

From (13) and (14) we have

$$
P \nu=Q \nu=R \nu=T \nu=\nu .
$$

This implies $\nu$ is a common fixed point of $\mathrm{P}, \mathrm{R}, \mathrm{Q}$ and $\mathrm{T}$.

\section{UNIQUENESS}

Suppose $\eta(\nu \neq \eta)$ is one more common fixed point of $\mathrm{P}, \mathrm{Q}, \mathrm{R}$, and $\mathrm{T}$.

Then $P \eta=T \eta=R \eta=Q \eta=\eta$.

Put $\alpha=\eta$ and $\beta=\nu$ in (3.1.2) we have

$$
\begin{aligned}
S(P \eta, P \eta, R \nu) \leq \lambda \max & \{S(Q \eta, Q \eta, T \nu), S(R \nu, R \nu, P \eta), \\
& \left.\frac{S(P \eta, P \eta, Q \eta) S(R \nu, R \nu, T \nu)}{S(Q \eta, Q \eta, T \nu)}\right\}
\end{aligned}
$$

in turn implies

$$
\begin{array}{r}
S(\eta, \eta, \nu) \leq \lambda \max \{S(\eta, \eta, \nu), S(\nu, \nu, \eta), \\
\left.\frac{S(\eta, \eta, \eta) S(\nu, \nu, \nu)}{S(\eta, \eta, \nu)}\right\}
\end{array}
$$

this in turn implies thus

$$
\eta=\nu
$$

This ensures that the common fixed point is unique.

\section{Now we present another theorem.}

\subsection{Theorem}

Let $\mathrm{P}, \mathrm{R}, \mathrm{Q}$ and $\mathrm{T}$ be four self maps in $\mathrm{S}$-metric space $(X, S)$ into itself holding the conditions

(3.1.1) ,(3.1.2),(3.1.4) , (3.1.5) .Further, the pairs (P,Q ) and ( R,T) are sub compatible and subsequentially continuous. then the above mappings will have a unique common fixed point.

Proof:

Since the pairs (P,Q ) and ( R,T) are sub compatible and sub sequentially continuous, therefore, $\exists$ two sequences $\left\{\alpha_{k}\right\}$ and $\left\{\beta_{k}\right\} \in X$ such that $\lim _{k \rightarrow \infty} P \alpha_{k}=\lim _{k \rightarrow \infty} Q \alpha_{k}=u$ for some $u \in X$ and which satisfy $\lim _{k \rightarrow \infty} S\left(P Q \alpha_{k}, Q P \alpha_{k}, z\right)=$ $S(P u, Q u, z)=1$ for all $z>0$.

$\lim _{k \rightarrow \infty} R \beta_{k}=\lim _{k \rightarrow \infty} T \beta_{k}=v$ for some $v \in X$ and which satisfy $\lim _{k \rightarrow \infty} S\left(R T \beta_{k}, T R \beta_{k}, z\right)=S(R v, T v, z)=1$ for all $z>0$. Therefore, $\mathrm{Pu}=\mathrm{Qu}$ and $\mathrm{Rv}=\mathrm{Tv}$. i.e., $\mathrm{u}$ is the coincidence point of $\mathrm{P}$ and $\mathrm{Q}$ and $\mathrm{v}$ is a coincidence point of $\mathrm{R}$ and $\mathrm{T}$.

Put $\alpha=\alpha_{k}$ and $\beta=\beta_{k}$ in (3.1.2) implies

$$
\begin{aligned}
& S\left(P \alpha_{k}, P \alpha_{k}, R \beta_{k}\right) \\
& \leq \lambda \max \left\{S\left(Q \alpha_{k}, Q \alpha_{k}, T \beta_{k}\right)\right. \\
& \left.S\left(R \beta_{k}, R \beta_{k}, P \alpha_{k}\right), \frac{S\left(P \alpha_{k}, P \alpha_{k}, Q \alpha_{k}\right) S\left(R \beta_{k}, R \beta_{k}, T \beta_{k}\right)}{S\left(Q \alpha_{k}, Q \alpha_{k}, T \beta_{k}\right)}\right\}
\end{aligned}
$$

$$
\begin{aligned}
S(u, u, v) \leq \lambda \max & \{S(u, u, v), S(v, v, u), \\
& \left.\frac{S(u, u, u) S(v, v, v)}{S(u, u, v)}\right\}
\end{aligned}
$$

$\Longrightarrow u=v$

Again put $\alpha=u$ and $\beta=\beta_{k}$ in (3.1.2) implies

$$
\begin{aligned}
S\left(P u, P u, R \beta_{k}\right) & \leq \lambda \max \\
& \left\{S\left(Q u, Q u, T \beta_{k}\right), S\left(R \beta_{k}, R \beta_{k}, P u\right),\right. \\
& \left.\frac{S(P u, P u, Q u) S\left(R \beta_{k}, R \beta_{k}, T \beta_{k}\right)}{S\left(Q u, Q u, T \beta_{k}\right)}\right\}
\end{aligned}
$$

This gives

$$
\begin{aligned}
& S(P u, P u, v) \leq \lambda \max \{S(P u, P u, v) \\
&\left.S(v, v, P u), \frac{S(P u, P u, P u) S(v, v, v)}{S(P u, P u, v)}\right\}
\end{aligned}
$$

$\Longrightarrow P u=v$

Therefore

$$
P u=Q u=u .
$$


Put $\alpha=\alpha_{k}$ and $\beta=v$ in (3.1.2) implies

$S\left(P \alpha_{k}, P \alpha_{k}, R v\right) \leq \lambda \max \left\{S\left(Q \alpha_{k}, Q \alpha_{k}, T v\right), S\left(R v, R v, P \alpha_{k}\right)\right.$ $\left.\frac{S\left(P \alpha_{k}, P \alpha_{k}, Q \alpha_{k}\right) S(R v, R v, T v)}{S\left(Q \alpha_{k}, Q \alpha_{k}, T v\right)}\right\}$

This implies

$$
\begin{aligned}
S(u, u, R v) \leq \lambda \max & \{S(u, u, R v), S(R v, R v, u), \\
& \left.\frac{S(u, u, u) S(R v, R v, T v)}{S(u, u, T v)}\right\} \because R v=T v
\end{aligned}
$$$$
\Longrightarrow R v=v
$$

$$
\therefore R v=T v=v \text {. }
$$

From (16)and(17)

$$
P u=Q u=R u=T u=u \because u=v
$$

Therefore $u$ is a common fixed point of $\mathrm{P}, \mathrm{R}, \mathrm{Q}$ and $\mathrm{T}$.

The uniqueness follows easily.

\section{EXAMPLE:}

Suppose $\mathrm{X}=[0,1]$ is a S-metric space with metrics $\delta_{1}$ and $\delta_{2}$ on $\mathrm{X}$ defind by $S(\alpha, \beta, \gamma)=\delta_{1}(\alpha, \gamma)+\delta_{2}(\beta, \gamma)$.where $\alpha, \beta, \gamma \in X$ and define mappings $\mathrm{P}, \mathrm{R}, \mathrm{Q}$ and $\mathrm{T}$ by

$$
\begin{aligned}
& P \alpha=\left\{\begin{array}{ll}
\frac{1-\alpha}{\frac{2}{2}} & \text { if } 0 \leq \alpha \leq \frac{1}{3}
\end{array} \text { if } \frac{1}{3}<\alpha \leq 1 .\right. \\
& R \alpha= \begin{cases}\frac{2 \alpha+1}{5} & \text { if } 0 \leq \alpha \leq \frac{1}{3} ; \\
\frac{2+\alpha}{7} & \text { if } \frac{1}{3}<\alpha \leq 1 .\end{cases} \\
& Q \alpha= \begin{cases}\alpha & \text { if } 0 \leq \alpha \leq \frac{1}{3} ; \\
\frac{1+\alpha}{2} & \text { if } \frac{1}{3}<\alpha \leq 1 .\end{cases} \\
& \text { and } T \alpha= \begin{cases}\frac{1+\alpha}{4} & \text { if } 0 \leq \alpha \leq \frac{1}{3} ; \\
1-\alpha & \text { if } \frac{1}{3}<\alpha \leq 1 .\end{cases}
\end{aligned}
$$

Now $\mathrm{P}(\mathrm{X})=\left[\frac{1}{5}, \frac{1}{2}\right] \mathrm{Q}(\mathrm{X})=[0,1]$ and $\mathrm{R}(\mathrm{X})=\left[\frac{1}{5}, \frac{3}{7}\right], \mathrm{T}(\mathrm{X})=\left[0, \frac{2}{3}\right)$.

this shows that $P(X) \subseteq T(X)$ and $R(X) \subseteq Q(X)$

Now, Let $\left\{\alpha_{k}\right\}$ as $\alpha_{k}=\frac{1}{3}-\frac{1}{k}$ for $k \geq 0$.

Now $P \alpha_{k}=P\left(\frac{1}{3}-\frac{1}{k}\right)=\left(\frac{1-\frac{1}{3}-\frac{1}{k}}{2}\right)=\frac{1}{3}+\frac{1}{2 k}=\frac{1}{3}$ and $Q\left(\alpha_{k}\right)=Q\left(\frac{1}{3}-\frac{1}{k}\right)=\left(\frac{1}{3}-\frac{1}{k}\right)=\frac{1}{3}$ as $k \rightarrow \infty$.

Therefore $P\left(\alpha_{k}\right)=Q\left(\alpha_{k}\right)=\frac{1}{3}=\nu($ say $)$ as $k \rightarrow \infty$. Also $P(\nu)=P\left(\frac{1}{3}\right)=\frac{1}{3}$ and $Q(\nu)=Q\left(\frac{1}{3}\right)=\frac{1}{3}$.

Further $P P\left(\alpha_{k}\right)=P P\left(\frac{1}{3}-\frac{1}{k}\right)=P\left(\frac{1-\left(\frac{1}{3}-\frac{1}{k}\right.}{2}\right)=$ $P\left(\frac{1}{3}+\frac{1}{2 k}\right)=\frac{2-\left(\frac{1}{3}+\frac{1}{2 k}\right)}{2}=\frac{1}{3}-\frac{1}{4 k}=\frac{1}{3}$ and $P Q\left(\alpha_{k}\right)=P Q\left(\frac{1}{3}-\frac{1}{k}\right)=P\left(\frac{1}{3}-\frac{1}{k}\right)=\frac{1-\left(\frac{1}{3}-\frac{1}{k}\right)}{2}=$ $\frac{1}{3}-\frac{1}{2 k}=\frac{1}{3}$ as $k \rightarrow \infty$.

Hence $P P\left(\alpha_{k}\right)=P Q\left(\alpha_{k}\right)=Q \nu$ as $k \rightarrow \infty$.

Similarly $Q Q\left(\alpha_{k}\right)=Q Q\left(\frac{1}{3}-\frac{1}{k}\right)=Q\left(\frac{1}{3}-\frac{1}{k}\right)=$ $Q\left(\frac{1}{3}-\frac{1}{k}\right)=\frac{1}{3}-\frac{1}{k}=\frac{1}{3}$ and

$Q P\left(\alpha_{k}\right)=Q P\left(\frac{1}{3}-\frac{1}{k}\right)=Q\left(\frac{1-\left(\frac{1}{3}-\frac{1}{k}\right)}{2}\right)=Q\left(\frac{1}{3}-\frac{1}{2 k}\right)$ $=\frac{1}{3}$ as $k \rightarrow \infty$.

Hence $Q Q\left(\alpha_{k}\right)=Q P\left(\alpha_{k}\right)=P \nu$ as $k \rightarrow \infty$. $\therefore S\left(P^{2} \alpha_{k}, P Q \alpha_{k}, Q \nu\right)=S\left(\frac{1}{3}, \frac{1}{3}, \frac{1}{3}\right)=0$ and $S\left(Q^{2} \alpha_{k}, Q P \alpha_{k}, P \nu\right)=S\left(\frac{1}{3}, \frac{1}{3}, \frac{1}{3}\right)=0$ as $k \rightarrow \infty$.

Hence the pair $(P, Q)$ is compatible of type -E.

Now $R \alpha_{k}=R\left(\frac{1}{3}-\frac{1}{k}\right)=\frac{2\left(\frac{1}{3}-\frac{1}{k}\right)+1}{5}=\frac{1}{3}-\frac{2}{5 k}=\frac{1}{3}$

and $T\left(\alpha_{k}\right)=T\left(\frac{1}{3}-\frac{1}{k}\right)=\frac{1+\left(\frac{1}{3}-\frac{1}{k}\right)}{4}=\frac{1}{3}$ as $k \rightarrow \infty$. $\therefore R\left(\alpha_{k}\right)=T\left(\alpha_{k}\right)=\frac{1}{3}=\nu($ say $)$ as $k \rightarrow \infty$.

Also $R(\nu)=R\left(\frac{1}{3}\right)=\frac{1}{3}$ and $T(\nu)=T\left(\frac{1}{3}\right)=\frac{1}{3}$. $=$

Further $R R\left(\alpha_{k}\right)=R R\left(\frac{1}{3}-\frac{1}{k}\right)=R\left(\frac{2\left(\frac{1}{3}-\frac{1}{k}\right)+1}{5}\right)$

$=R\left(\frac{1}{3}-\frac{2}{5 k}\right) \frac{2\left(\frac{1}{3}-\frac{2}{5 k}\right)+1}{5}=\frac{1}{3}-\frac{4}{25 k}=\frac{1}{3}$, and

$R T\left(\alpha_{k}\right)=R T\left(\frac{1}{3}-\frac{1}{k}\right)=R\left(\frac{1}{3}+\frac{1}{4 k}\right)=\frac{2+\left(\frac{1}{3}+\frac{1}{2 k}\right)}{7}$

$=\frac{1}{3}$ as $k \rightarrow \infty$.

Hence $R R\left(\alpha_{k}\right)=R T\left(\alpha_{k}\right)=Q \nu$ as $k \rightarrow \infty$.

Similarly $T T\left(\alpha_{k}\right)=T T\left(\frac{1}{3}-\frac{1}{k}\right)=T \frac{\left(1-\left(\frac{1}{3}-\frac{1}{k}\right)\right.}{4}=$ $T\left(\frac{1}{3}-\frac{1}{4 k}\right)=\frac{2-\left(\frac{1}{3}-\frac{1}{4 k}\right)}{5}=\frac{1}{3}$ and

$T R\left(\alpha_{k}\right)=T R\left(\frac{1}{3}-\frac{1}{k}\right)=T\left(\frac{2\left(\frac{1}{3}-\frac{1}{k}\right)+1}{5}\right)=T\left(\frac{1}{3}-\frac{2}{5 k}\right)$ $=\frac{1}{3}$ as $k \rightarrow \infty$.

Hence $T T\left(\alpha_{k}\right)=T R\left(\alpha_{k}\right)=R \nu$ were $k \rightarrow \infty$.

$\therefore S\left(R^{2} \alpha_{k}, R T \alpha_{k}, Q \nu\right)=S\left(\frac{1}{3}, \frac{1}{3}, \frac{1}{3}\right)=0$ and

$S\left(T^{2} \alpha_{k}, T R \alpha_{k}, R \nu\right)=S\left(\frac{1}{3}, \frac{1}{3}, \frac{1}{3}\right)=0$ as $k \rightarrow \infty$.

Hence the pair $(R, T)$ is compatible of type -E.

Also $S\left(P Q \alpha_{k}, P Q \alpha_{k}, P \nu\right)=S\left(\frac{1}{3}, \frac{1}{3}, \frac{1}{3}\right)=0$ and $S\left(Q P \alpha_{k}, Q P \beta_{k}, Q \nu\right)=S\left(\frac{1}{3}, \frac{1}{3}, \frac{1}{3}\right)=0$ when $k \rightarrow \infty$.

Hence the pair $(P, Q)$ is sub sequentially continuous mapping.

Also $S\left(R T \alpha_{k}, R T \alpha_{k}, R \nu\right)=S\left(\frac{1}{3}, \frac{1}{3}, \frac{1}{3}\right)=0$ and

$S\left(T R \alpha_{k}, T R \alpha_{k}, T \nu\right)=S\left(\frac{1}{3}, \frac{1}{3}, \frac{1}{3}\right)=0$ as $k \rightarrow \infty$.

Therefore the pair $(R, T)$ is sub sequentially continuous mapping. Thus the pairs $(\mathrm{P}, \mathrm{Q})$ and $(\mathrm{R}, \mathrm{T})$ are compatible mappings of type-E and sub sequentially continuous.

We now verify the condition (3.1.2).

\section{Case-1}

If $\alpha, \beta \in\left[0, \frac{1}{3}\right]$, then $S(\alpha, \beta, \gamma)=\delta_{1}(\alpha, \gamma)+\delta_{2}(\beta, \gamma)$

Let $\alpha=\frac{1}{4}, \beta=\frac{1}{5}$ in (3.1.2) we get

$$
\begin{aligned}
S\left(\frac{2}{5}, \frac{2}{5}, \frac{7}{25}\right) \leq \lambda \max & \left\{S\left(\frac{1}{4}, \frac{1}{4}, \frac{5}{16}\right), S\left(\frac{7}{25}, \frac{7}{25}, \frac{2}{5}\right),\right. \\
& \left.\frac{S\left(\frac{2}{5}, \frac{2}{5}, \frac{1}{4}\right) S\left(\frac{7}{25}, \frac{7}{25}, \frac{2}{5}\right)}{S\left(\frac{1}{4}, \frac{1}{4}, \frac{5}{16}\right)}\right\}
\end{aligned}
$$

$0.24 \leq \lambda \max \left\{0.125,0.24, \frac{0.3 \times 0.24}{0.125}\right\}$

Hence

$0.24 \leq \lambda \max \{0.125,0.24,0.576\}$

$0.24 \leq \lambda(0.576) \Longrightarrow \lambda=0.417 \in(0,1)$,

as a result, inequality(3.1.2) is true.

\section{Case-2}

If $\alpha, \beta \in\left(\frac{1}{3}, 1\right]$, then $S(\alpha, \beta, \gamma)=\delta_{1}(\alpha, \gamma)+\delta_{2}(\beta, \gamma)$

Let $\alpha=\frac{1}{2}, \beta=\frac{2}{3}$ in (3.1.2) we get

$$
\begin{aligned}
S\left(\frac{4}{15}, \frac{4}{15}, \frac{8}{21}\right) \leq \lambda \max & \left\{S\left(\frac{3}{4}, \frac{3}{4}, \frac{1}{2}\right), S\left(\frac{8}{21}, \frac{8}{21}, \frac{4}{15}\right),\right. \\
& \left.\frac{S\left(\frac{4}{15}, \frac{4}{15}, \frac{3}{4}\right) S\left(\frac{8}{21}, \frac{8}{21}, \frac{1}{2}\right)}{S\left(\frac{3}{4}, \frac{3}{4}, \frac{1}{2}\right)}\right\}
\end{aligned}
$$

$0.23 \leq \lambda \max \left\{0.5,0.23, \frac{0.96 X .23}{0.5}\right\}$

Thus we have $0.23 \leq \lambda \max \{0.5,0.23,0.44\}$ 
$0.23 \leq \lambda(0.5) \Longrightarrow \lambda=0.46 \in(0,1)$, as a result ,the inequality (3.1.2) is true.

The remaining cases can be verified in the same way.

\section{CONCLUSION}

This paper dealt with the generation of two fixed point theorems. In the first theorem, both pairs of the mappings are assumed to be sub sequentially continuous mappings and compatible of type-E. In the second theorem both the pairs are assumed to be sub sequentially continuous and sub -compatible mappings. Further, the mappings in our results are not assumed to be continuous. Note that the result of this article generalize the results of $\{[1],[7],[9],[10],[11]$ and $[12]\}$ using weaker conditions sub -sequentially continuous mappings, sub compatible mappings instead of continuous mappings, weakly compatible, occasionally weakly compatible, reciprocally continuous and weakly reciprocally continuous mappings. Moreover, our findings are confirmed by appropriate examples.

\section{REFERENCES}

[1] Sedghi, Shaban, Nabi Shobe, and Abdelkrim Aliouche. "A generalization of fixed point theorems in S-metric spaces." Matematički vesnik 64.3 , pp. 258-266,2012.

[2] Singh M. R., and Y. Mahendra Singh. "Compatible mappings of type (E) and common fixed point theorems of Meir-Keeler type.” Int. J. Math. Sci. Engg. App,1 1.2 ,pp 299-315,2007. https://www.researchgate.net/publication/266188444.

[3] Godet-Thobie C., and B. Satco. "Decomposability and uniform integrability in Pettis integration." Quaestiones Mathematicae 29.1, pp 39-58,2006.DOI:10.2989/16073600609486148.
[4] Beloul, Said. "Some fixed point theorems for weakly subsequentially continuous and compatible of type (E) mappings with an application." International Journal of Nonlinear Analysis and Applications 7.1 pp 53-62,2016, DOI:10.22075/ijnaa.2015.294.

[5] Sharma, Rajinder, Vishal Gupta, and Mukesh Kushwaha. ”New Results for Compatible Mappings of Type A and Subsequential Continuous Mappings." Applications and Applied Mathematics: An International Journal (AAM), 15.1, 16,pp 282295,2020.http://pvamu.edu/aam.

[6] R. P. Pant, "Common fixed points of four mappings," Bulletin of the Calcutta Mathematical Society, vol. 90, no. 4,pp 281-286.1998.

[7] Srinivas V., and K. Mallaiah. "Some results on a weaker class of compatible mappings in S-metric space." Malaya Journal of Matematik (MJM) 8.3, pp 1132-1137,2020.

[8] Bouhadjera, Hakima. "More general common fixed point theorems under a new concept." Demonstratio Mathematica,49.1 pp 6478,2016.DOI:10.1515/dema-2016-0007.

[9] Mallaiah K., and V. Srinivas. "Some results on S-metric space." Advances in Mathematics: Scientific Journal 9 (2020), no.9, 7663-7677.

[10] Jong Kyu Kim, Shaban Sedghi, A. Gholidahneh, and M. Mahdi Rezaee "Fixed point theorems in S-metric space" East Asian Math. J.Vol. 32 (2016), No. 5, pp. 677-684.

[11] Beny J., S. Jone Jayashree, and P. Sagaya Leeli. "A study of fixed point theorems in S-metric space." European Journal of Molecular \& Clinical Medicine 7.9 (2021): 3197-3203.

[12] Chaipornjareansri, Somkiat. "Fixed Point Theorems for Generalized Weakly Contractive Mappings in S-Metric Space." Thai Journal of Mathematics (2018): 50-62. 